\title{
Pseudo Harmonic Morphisms on Riemannian Polyhedra
}

\author{
M. A. Aprodu, T. Bouziane
}

\begin{abstract}
The aim of this paper is to extend the notion of pseudo harmonic morphism (introduced by Loubeau [13]) to the case when the source manifold is an admissible Riemannian polyhedron. We define these maps to be harmonic in the sense of EellsFuglede [7] and pseudo-horizontally weakly conformal in our sense (see Section 3). We characterize them by means of germs of harmonic functions on the source polyhedron, in sense of Korevaar-Schoen [11], and germs of holomorphic functions on the Kähler target manifold.

Keywords and phrases: Harmonic maps, Harmonic morphisms, Riemannian polyhedra, PHWC maps, PHM maps, Kähler manifolds.
\end{abstract}

\section{Introduction.}

"Harmonicity" is a topic which is situated between geometry and analysis. For instance, Fuglede [8] and Ishihara [10], independently, proved that harmonic morphisms between smooth Riemannian manifolds (maps which pull back germs of harmonic functions to germs of harmonic functions) are precisely harmonic maps (analytic property) which are horizontally weakly conformal (geometric property). A natural question arises: is there any equivalent notion if the target manifolds are Hermitian or Kähler? If yes, can we characterize geometrically this notion? Loubeau, in [13], gave complete answers to these questions and named the maps "Pseudo harmonic morphism".

In [11], Korevaar and Schoen extended the theory of harmonic maps between smooth Riemannian manifolds to the case of maps between certain singular spaces: for example

M. A. Aprodu: Department of Mathematics, University of Galaţi, Domnească Str. 47, RO-6200, Galaţi, Romania.

e-mail: Monica.Aprodu@ugal.ro

T. Bouziane: The Abdus Salam International Center for Theoretical Physics, strada Costiera 11, 34014 Trieste, Italy.

e-mail: tbouzian@ictp.trieste.it

Mathematics Subject Classification (2000): 58E20, 53C43, 53C55, 32Q15. 
admissible Riemannian polyhedra. The Riemannian polyhedra are both very interesting examples of the "geometric habitat" of the harmonicity (being harmonic spaces) and provide several examples: smooth Riemannian manifolds, Riemannian orbit spaces, normal analytic spaces, Thom spaces etc. Later, Eells and Fuglede in [7], expanded the notion of harmonic morphisms to the case of Riemannian polyhedra. But, to give the same characterization for harmonic morphisms between Riemannian polyhedra as Fuglede and Ishihara did in the smooth case, they had to pay a price: the target had to be a smooth Riemannian manifold. Also, many of the properties found for the harmonic maps and harmonic morphisms in the smooth case could be recovered when consider as domain and target Riemannian polyhedra.

Remaining in the same spirt of ideas, the aim of this paper is to extend pseudo harmonic morphisms to the case when the domain is an admissible Riemannian polyhedron and the target a Kähler manifold and to characterize them (as it was done in the smooth case) by "geometric criteria" and "analytic criteria". It turns out, because of the absence of global differential calculus on singular spaces, that it is not easy to find a good definition of the pseudo harmonic morphisms on Riemannian polyhedra generalizing in a natural way the smooth case. Another difficulty, compare with Loubeau's results, is to find a geometric condition which characterize pseudo harmonic morphisms on Riemannian polyhedra, knowing that we can not talk about horizontal vectors for example. A third difficulty is in the use of germs of harmonic functions in the sense of Korevaar-Schoen, as the analytic aspect of our construction.

The outline of the paper is as follows. In Section 2 we recall some results on Riemannian polyhedra, harmonic maps and morphisms between Riemannian polyhedra. Section 3 is devoted to the (alternative) geometric characterization of the pseudo harmonic morphisms defined on Riemannian polyhedra named (also) " pseudo-horizontally weak conformality". We show that this geometric property is preserved by the holomorphy. In Section 4, we introduce the notion of pseudo harmonic morphisms from a Riemannian polyhedra to a Kähler manifold and characterize them as maps which pull back germs of holomorphic functions on target manifold to germs of harmonic functions on the Riemannian polyhedron (Theorem 4.2). We also state a lifting property for pseudo harmonic morphisms (Proposition 4.7).

Finally, in Section 5, applying Proposition 4.7 we give some interesting examples.

\section{Preliminaries.}

This section is devoted to some basic notions and known results which will be used in the next sections.

\subsection{Riemannian polyhedra.}

\subsubsection{Riemannian admissible complexes ([3], [4], [5], [6], [16]).}

Let $K$ be a locally finite simplicial complex, endowed with a piecewise smooth Riemannian metric $g$ (i.e. $g$ is a family of smooth Riemannian metrics $g_{\Delta}$ on simplices $\Delta$ of $K$, such that the restriction $\left(g_{\Delta}\right)_{\mid \Delta^{\prime}}=g_{\Delta^{\prime}}$, for any simplices $\Delta^{\prime}$ and $\Delta$ with $\Delta^{\prime} \subset \Delta$ ). 
Let $K$ be a finite dimensional simplicial complex which is connected locally finite. A map $f$ from $[a, b]$ to $K$ is called a broken geodesic if there is a subdivision $a=t_{0}<t_{1}<$ $\ldots<t_{p+1}=b$, such that $f\left(\left[t_{i}, t_{i+1}\right]\right)$ is contained in some cell and the restriction of $f$ to $\left[t_{i}, t_{i+1}\right]$ is a geodesic inside that cell. Then define the length of the broken geodesic map $f$ to be:

$$
L(f)=\sum_{i=0}^{p} d\left(f\left(t_{i}\right), f\left(t_{i+1}\right)\right) .
$$

The length inside each cell being measured with respect to its metric.

Then, define $\tilde{d}(x, y)$, for every two points $x, y$ in $K$, to be the lower bound of the lengths of broken geodesics from $x$ to $y$. $\tilde{d}$ is a pseudo-distance.

If $K$ is connected and locally finite, then $(K, \tilde{d})$ is a length space and hence a geodesic space (i.e. a metric space where every two points are connected by a curve with length equal to the distance between them ) if complete.

A $l$-simplex in $K$ is called a boundary simplex if it is adjacent to exactly one $l+1$ simplex. The complex $K$ is called boundaryless if there are no boundary simplices in $K$.

The (open) star of an open simplex $\Delta^{o}$ (i.e. the topological interior of $\Delta$ or the points of $\Delta$ not belonging to any sub-face of $\Delta$; if $\Delta$ is point then $\Delta^{o}=\Delta$ ) of $K$ is defined as:

$$
\operatorname{st}\left(\Delta^{o}\right)=\bigcup\left\{\Delta_{i}^{o}: \Delta_{i} \text { is simplex of } K \text { with } \Delta_{i} \supset \Delta\right\} .
$$

The star $s t(p)$ of point $p$ is defined as the star of its carrier, the unique open simplex $\Delta^{o}$ containing $p$. Every star is path connected and contains the star of its points. In particular $K$ is locally path connected. The closure of any star is sub-complex.

We say that the complex $K$ is admissible, if it is dimensionally homogeneous and for every connected open subset $U$ of $K$, the open set $U \backslash\{U \cap\{$ the $(k-2)-$ skeleton $\}$ \} is connected, where $k$ is the dimension of $K$ (i.e. $K$ is $(n-1)$-chainable).

Let $x \in K$ a vertex of $K$ so that $x$ is in the $l$-simplex $\Delta_{l}$. We view $\Delta_{l}$ as an affine simplex in $\mathbf{R}^{l}$, that is $\Delta_{l}=\bigcap_{i=0}^{l} H_{i}$, where $H_{0}, H_{1}, \ldots, H_{l}$ are closed half spaces in general position, and we suppose that $x$ is in the topological interior of $H_{0}$. The Riemannian metric $g_{\Delta_{l}}$ is the restriction to $\Delta_{l}$ of a smooth Riemannian metric defined in an open neighborhood $V$ of $\Delta_{l}$ in $\mathbf{R}^{l}$. The intersection $T_{x} \Delta_{l}=\bigcap_{i=1}^{l} H_{i} \subset T_{x} V$ is a cone with apex $0 \in T_{x} V$, and $g_{\Delta_{l}}(x)$ turns it into an Euclidean cone. Let $\Delta_{m} \subset \Delta_{l}(m<l)$ be another simplex adjacent to $x$. Then, the face of $T_{x} \Delta_{l}$ corresponding to $\Delta_{m}$ is isomorphic to $T_{x} \Delta_{m}$ and we view $T_{x} \Delta_{m}$ as a subset of $T_{x} \Delta_{l}$.

Set $T_{x} K=\bigcup_{\Delta_{i} \ni x} T_{x} \Delta_{i}$ and we call it the tangent cone of $K$ at $x$. Let $S_{x} \Delta_{l}$ denote the subset of all unit vectors in $T_{x} \Delta_{l}$ and set $S_{x}=S_{x} K=\bigcup_{\Delta_{i} \ni x} S_{x} \Delta_{i}$. The set $S_{x}$ is called the link of $x$ in $K$. If $\Delta_{l}$ is a simplex adjacent to $x$, then $g_{\Delta_{l}}(x)$ defines a Riemannian metric on the $(l-1)$-simplex $S_{x} \Delta_{l}$. The family $g_{x}$ of riemannian metrics $g_{\Delta_{l}}(x)$ turns $S_{x} \Delta_{l}$ into a simplicial complex with a piecewise smooth Riemannian metric such that the simplices are spherical.

We call an admissible connected locally finite simplicial complex, endowed with a piecewise smooth Riemannian metric, an admissible Riemannian complex. 


\subsubsection{Riemannian polyhedron [7].}

We mean by polyhedron a connected locally compact separable Hausdorff space $X$ for which there exists a simplicial complex $K$ and homeomorphism $\theta: K \rightarrow X$. Any such pair $(K, \theta)$ is called a triangulation of $X$. The complex $K$ is necessarily countable and locally finite (cf. [15] page 120) and the space $X$ is path connected and locally contractible. The dimension of $X$ is by definition the dimension of $K$ and it is independent of the triangulation.

A sub-polyhedron of a polyhedron $X$ with given triangulation $(K, \theta)$, is the polyhedron $X^{\prime} \subset X$ having as a triangulation $\left(K^{\prime}, \theta_{\mid K^{\prime}}\right)$ where $K^{\prime}$ is a subcomplex of $K$ (i.e. $K^{\prime}$ is the complex whose vertices and simplexes are some of those of $K$ ).

If $X$ is a polyhedron with specified triangulation $(K, \theta)$, we shall speak of vertices, simplexes, $i$-skeletons or stars of $X$ respectively of a space of links or tangent cones of $X$ as the image under $\theta$ of vertices, simplexes, $i$-skeletons or stars of $K$ respectively the image of space of links or tangent cones of $K$. Thus our simplexes become compact subsets of $X$ and the $i$-skeletons and stars become sub-polyhedrons of $X$.

If for given triangulation $(K, \theta)$ of the polyhedron $X$, the homeomorphism $\theta$ is locally bi-lipschitz then $X$ is said Lip polyhedron and $\theta$ Lip homeomorphism.

A null set in a Lip polyhedron $X$ is a set $Z \subset X$ such that $Z$ meets every maximal simplex $\Delta$, relative to a triangulation $(K, \theta)$ (hence any) in set whose pre-image under $\theta$ has $n$-dimensional Lebesgue measure $0, n=\operatorname{dim} \Delta$. Note that 'almost everywhere' (a.e.) means everywhere except in some null set.

A Riemannian polyhedron $X=(X, g)$ is defined as a Lip polyhedron $X$ with a specified triangulation $(K, \theta)$ such that $\mathrm{K}$ is a simplicial complex endowed with a covariant bounded measurable Riemannian metric tensor $g$, satisfying the ellipticity condition below. In fact, suppose that $X$ has homogeneous dimension $n$ and choose a measurable riemannian metric $g_{\Delta}$ on the open euclidean $n$-simplex $\theta^{-1}\left(\Delta^{o}\right)$ of $K$. In terms of euclidean coordinates $\left\{x_{1}, \ldots, x_{n}\right\}$ of points $x=\theta^{-1}(p), g_{\Delta}$ thus assigns to almost every point $p \in \Delta^{o}$ (or $\left.x\right)$, an $n \times n$ symmetric positive definite matrix $g_{\Delta}=\left(g_{i j}^{\Delta}(x)\right)_{i, j=1, \ldots, n}$ with measurable real entries and there is a constant $\Lambda_{\Delta}>0$ such that (ellipticity condition):

$$
\Lambda_{\Delta}^{-2} \sum_{i=0}^{n}\left(\xi^{i}\right)^{2} \leqslant \sum_{i, j} g_{i j}^{\Delta}(x) \xi^{i} \xi^{j} \leqslant \Lambda_{\Delta}^{2} \sum_{i=0}^{n}\left(\xi^{i}\right)^{2}
$$

for a.e. $x \in \theta^{-1}\left(\Delta^{o}\right)$ and every $\xi=\left(\xi^{1}, \ldots, \xi^{n}\right) \in \mathbf{R}^{n}$. This condition amounts to the components of $g_{\Delta}$ being bounded and it is independent not only of the choice of the euclidean frame on $\theta^{-1}\left(\Delta^{o}\right)$ but also of the chosen triangulation.

For simplicity of statements we shall sometimes require that, relative to a fixed triangulation $(K, \theta)$ of Riemannian polyhedron $X$ (uniform ellipticity condition),

$$
\Lambda:=\sup \left\{\Lambda_{\Delta}: \Delta \text { is simplex of } X\right\}<\infty .
$$

A Riemannian polyhedron $X$ is said to be admissible if for a fixed triangulation $(K, \theta)$ (hence any) the Riemannian simplicial complex $K$ is admissible.

We underline that (for simplicity) the given definition of a Riemannian polyhedron $(X, g)$ contains already the fact (because of the definition above of the Riemannian admissible complex) that the metric $g$ is continuous relative to some (hence any) triangulation (i.e. for every maximal simplex $\Delta$ the metric $g_{\Delta}$ is continuous up to the boundary). 
This fact is sometimes in the literature omitted. The polyhedron is said to be simplexwise smooth if relative to some triangulation $(K, \theta)$ (and hence any), the complex $K$ is simplexwise smooth. Both continuity and simplexwise smoothness are preserved under subdivision.

In the case of a general bounded measurable Riemannian metric $g$ on $X$, we often consider, in addition to $g$, the euclidean Riemannian metric $g^{e}$ on the Lip polyhedron $X$ with a specified triangulation $(K, \theta)$. For each simplex $\Delta, g_{\Delta}^{e}$ is defined in terms of euclidean frame on $\theta^{-1}\left(\Delta^{o}\right)$ as above by unit matrix $\left(\delta_{i j}\right)_{i, j}$. Thus $g^{e}$ is by no means covariantly defined and should be regarded as a mere reference metric on the triangulated polyhedron $X$.

Relative to a given triangulation $(K, \theta)$ of an $n$-dimensional Riemannian polyhedron $(X, g)$ (not necessarily admissible), we have on $X$ the distance function $e$ induced by the euclidean distance on the euclidean space $V$ in which $K$ is affinely Lip embedded. This distance $e$ is not intrinsic but it will play an auxiliary role in defining an equivalent distance $d_{X}$ as follows:

Let $\mathfrak{Z}$ denote the collection of all null sets of $X$. For given triangulation $(K, \theta)$ consider the set $Z_{K} \subset \mathfrak{Z}$ obtained from $X$ by removing from each maximal simplex $\Delta$ in $X$ those points of $\Delta^{o}$ which are Lebesgue points for $g_{\Delta}$. For $x, y \in X$ and any $Z \in \mathfrak{Z}$ such that $Z \subset Z_{K}$ we set:

$$
\begin{gathered}
d_{X}(x, y)=\sup _{Z \in \mathfrak{Z}} \quad \inf _{Z} \quad\left\{L_{K}(\gamma): \gamma \quad \begin{array}{cc}
\text { is Lip continuous path } \\
\text { and transversal to } Z
\end{array}\right\}, \\
\quad \gamma(a)=x \\
\gamma(b)=y
\end{gathered}
$$

where $L_{K}(\gamma)$ is the length of the path $\gamma$ defined as:

$$
L_{K}(\gamma)=\sum_{\Delta \subset X_{\gamma^{-1}\left(\Delta^{o}\right)}} \sqrt{\left(g_{i j}^{\Delta} \circ \theta^{-1} \circ \gamma \cdot\right) \gamma^{i} \gamma^{j}}, \quad \begin{aligned}
& \text { the sum is over } \\
& \text { all simplexes meeting } \gamma .
\end{aligned}
$$

It is shown in [7] that the distance $d_{X}$ is intrinsic, in particular it is independent of the chosen triangulation and it is equivalent to the euclidean distance $e$ (due to the Lip affinely and homeomorphically embedding of $X$ in some euclidean space $V$ ).

\subsection{Energy of maps}

The concept of energy in the case of a map of Riemannian domain into an arbitrary metric space $Y$ was defined and investigated by Korevaar and Schoen [11]. Later this concept was extended by Eells and Fuglede [7] to the case of map from an admissible Riemannian polyhedron $X$ with simplexwise smooth Riemannian metric. Thus, the energy $E(\varphi)$ of a map $\varphi$ from $X$ to the space $Y$ is defined as the limit of suitable approximate energy expressed in terms of the distance function $d_{Y}$ of $Y$.

It is shown in [7] that the maps $\varphi: X \rightarrow Y$ of finite energy are precisely those quasicontinuous (i.e. has a continuous restriction to closed sets), whose complements have arbitrarily small capacity, (cf. [7] page 153) whose restriction to each top dimensional simplex of $X$ has finite energy in the sense of Korevaar-Schoen, and $E(\varphi)$ is the sum of the energies of these restrictions. 
Now, let $(X, g)$ be an admissible $m$-dimensional Riemannian polyhedron with simplexwise smooth Riemannian metric. It is not required that $g$ is continuous across lower dimensional simplexes. The target $\left(Y, d_{Y}\right)$ is an arbitrary metric space.

Denote $L_{l o c}^{2}(X, Y)$ the space of all $\mu_{g}$-measurable ( $\mu_{g}$ the volume measure of $g$ ) maps $\varphi: X \rightarrow Y$ having separable essential range and for which the map $d_{Y}(\varphi(), q.) \in$ $L_{l o c}^{2}\left(X, \mu_{g}\right)$ (i.e. locally $\mu_{g}$-squared integrable) for some point $q$ (hence by triangle inequality for any point). For $\varphi, \psi \in L_{l o c}^{2}(X, Y)$ define their distance $D(\varphi, \psi)$ by:

$$
D^{2}(\varphi, \psi)=\int_{X} d_{Y}^{2}(\varphi(x), \psi(y)) d \mu_{g}(x) .
$$

Two maps $\varphi, \psi \in L_{l o c}^{2}(X, Y)$ are said to be equivalent if $D(\varphi, \psi)=0$, (i.e. $\varphi(x)=\psi(x)$ $\mu_{g}$-a.e.). If the space $X$ is compact then $D(\varphi, \psi)<\infty$ and $D$ is a metric on $L_{l o c}^{2}(X, Y)=$ $L^{2}(X, Y)$ and complete if the space $Y$ is complete [11].

The approximate energy density of the map $\varphi \in L_{l o c}^{2}(X, Y)$ is defined for $\epsilon>0$ by:

$$
e_{\epsilon}(\varphi)(x)=\int_{B_{X}(x, \epsilon)} \frac{d_{Y}^{2}\left(\varphi(x), \varphi\left(x^{\prime}\right)\right)}{\epsilon^{m+2}} d \mu_{g}\left(x^{\prime}\right) .
$$

The function $e_{\epsilon}(\varphi) \geqslant 0$ is locally $\mu_{g}$-integrable.

The energy $E(\varphi)$ of a map $\varphi$ of class $L_{l o c}^{2}(X, Y)$ is:

$$
E(\varphi)=\sup _{f \in C_{c}(X,[0,1])}\left(\limsup _{\epsilon \rightarrow 0} \int_{X} f e_{\epsilon}(\varphi) d \mu_{g}\right),
$$

where $C_{c}(X,[0,1])$ denotes the space of continuous functions from $X$ to the interval $[0,1]$ with compact support.

A map $\varphi: X \rightarrow Y$ is said to be locally of finite energy, and we write $\varphi \in W_{l o c}^{1,2}(X, Y)$, if $E\left(\varphi_{\mid U}\right)<\infty$ for every relatively compact domain $U \subset X$, or equivalently if $X$ can be covered by domains $U \subset X$ such that $E\left(\varphi_{\mid U}\right)<\infty$.

For example (cf. [7] Lemma 4.4), every Lip continuous map $\varphi: X \rightarrow Y$ is of class $W_{l o c}^{1,2}(X, Y)$. In the case when $X$ is compact $W_{l o c}^{1,2}(X, Y)$ is denoted $W^{1,2}(X, Y)$ the space of all maps of finite energy.

$W_{c}^{1,2}(X, Y)$ denotes the linear subspace of $W^{1,2}(X, Y)$ consisting of all maps of finite energy of compact support in $X$.

We can show (cf. [7] Theorem 9.1) that a real function $\varphi \in L_{l o c}^{2}(X)$ is locally of finite energy if and only if there is a function $e(\varphi) \in L_{l o c}^{1}(X)$, named energy density of $\varphi$, such that (weak convergence):

$$
\lim _{\epsilon \rightarrow 0} \int_{X} f e_{\epsilon}(\varphi) d \mu_{g}=\int_{X} f e(\varphi) d \mu_{g}, \text { for each } f \in C_{c}(X) .
$$

\subsection{Harmonic maps and harmonic morphisms on Riemannian poly- hedra [7].}

In this paragraph we shall remind some relevant results which give the relation between harmonic morphisms and harmonic maps on Riemannian polyhedra. 


\subsubsection{Harmonic maps.}

Let $(X, g)$ be an arbitrary admissible Riemannian polyhedron ( $g$ just bounded measurable with local elliptic bounds), $\operatorname{dim} X=m$ and $\left(Y, d_{Y}\right)$ a metric space .

A continuous map $\varphi: X \rightarrow Y$ of class $W_{l o c}^{1,2}(X, Y)$ is said to be harmonic if it is bi-locally E-minimizing, i.e. $X$ can be covered by relatively compact subdomains $U$ for each of which there is an open set $V \supset \varphi(U)$ in $Y$ such that

$$
E\left(\varphi_{\mid U}\right) \leqslant E\left(\psi_{\mid U}\right)
$$

for every continuous map $\psi \in W_{l o c}^{1,2}(X, Y)$, with $\psi(U) \subset V$ and $\psi=\varphi$ in $X \backslash U$.

Let $(N, h)$ denote a smooth Riemannian manifold without boundary, $\operatorname{dim}_{\mathbf{R}} N=n$ and $\Gamma_{\alpha \beta}^{k}$ the Christoffel symbols on $N$. By a weakly harmonic map $\varphi: X \rightarrow N$ we mean a quasicontinuous map (a map which is continuous on the complement of open sets of arbitrarily small capacity ; in the case of the Riemannian polyhedron $X$ it is just the complement of open subsets of the $(m-2)$-skeleton of $X)$ of class $W_{l o c}^{1,2}(X, N)$ with the following property:

For any chart $\eta: V \rightarrow \mathbf{R}^{n}$ on $\mathrm{N}$ and any quasiopen set $U \subset \varphi^{-1}(V)$ of compact closure in $\mathrm{X}$, the equation

$$
\int_{U}\left\langle\nabla \lambda, \nabla \varphi^{k}\right\rangle d \mu_{g}=\int_{U} \lambda\left(\Gamma_{\alpha \beta}^{k} \circ \varphi\right)\left\langle\nabla \varphi^{\alpha}, \nabla \varphi^{\beta}\right\rangle d \mu_{g},
$$

holds for every $k=1, \ldots, n$ and every bounded function $\lambda \in W_{0}^{1,2}(U)$.

It is shown in [7], (Theorem 12.1), that: for a continuous map $\varphi \in W_{l o c}^{1,2}(X, N)$ the following are equivalent:

(a) $\varphi$ is harmonic,

(b) $\varphi$ is weakly harmonic,

(c) $\varphi$ pulls convex functions on open sets $V \subset N$ back to subharmonic functions on $\varphi^{-1}(V)$.

\subsubsection{Harmonic morphisms.}

Denote by $X$ and $Y$ two Riemannian polyhedra (or any harmonic spaces in the sense of Brelot; see Chapter 2, [7]).

A continuous map $\varphi: X \rightarrow Y$ is a harmonic morphism if, for every open set $V \subset Y$ and for every harmonic function $v$ on $V, v \circ \varphi$ is harmonic on $\varphi^{-1}(V)$.

Let $\varphi: X \rightarrow Y$ be a nonconstant harmonic morphism, then (cf. [7], Theorem 13.1):

(i) $\varphi$ likewise pulls germs of superharmonic functions on $Y$ back to germs of superharmonic functions on $X$.

(ii) If $\varphi$ is surjective and proper then a function $v: V \rightarrow[-\infty, \infty](V$ open in $Y)$ is superharmonic [resp. harmonic] if (and only if) $v \circ \varphi$ is superharmonic [resp. harmonic] on $\varphi^{-1}(V)$.

Let $\left(N, g_{N}\right)$ denote a $n$-Riemannian manifold without boundary and suppose that the polyhedron $X$ is admissible. A continuous map $\varphi: X \rightarrow N$ of class $W_{l o c}^{1,2}(X, N)$ is called horizontally weakly conformal if there exist a scalar $\lambda$, defined a.e. in $X$, such that:

$$
\langle\nabla(v \circ \varphi), \nabla(w \circ \varphi)\rangle=\lambda\left[g_{N}\left(\nabla_{N} v, \nabla_{N} w\right) \circ \varphi\right] \text { a.e. in } \mathrm{X}
$$


for every pair of functions $v, w \in \mathcal{C}^{1}(N)$. Henceforth $\nabla_{N}$ denote the gradient operator on $N$ and $\nabla$ the gradient operator defined a.e. on the domain space $(X, g)$.

The property of horizontally weak conformality is a local one, thus it reads in terms of local coordinates $\left(y^{\alpha}\right)$ in $N$,

$$
\left\langle\nabla \varphi^{\alpha}, \nabla \varphi^{\beta}\right\rangle=\lambda\left(g_{N}^{\alpha \beta} \circ \varphi\right) \text { a.e. in } \mathrm{X}
$$

for $\alpha, \beta=1, \ldots, n$. Taking $\alpha=\beta, \lambda$ is uniquely determined and $\lambda \geqslant 0$ a.e. in $X$. Moreover, $\lambda \in L_{l o c}^{1}(X)$ because $\nabla \varphi^{\alpha} \in L_{l o c}^{1}(X)$. $\lambda$ is called the dilation of $\varphi$.

The notion of horizontally weak conformality is intimately related to the one of harmonic morphisms. For instance we can show (cf. [7], Theorem 13.2) that a continuous $\operatorname{map} \varphi: X \rightarrow N$ of class $W_{l o c}^{1,2}(X, N)$ is a harmonic morphism if and only if $\varphi$ is horizontally weakly conformal, harmonic map and equivalently, there is a scalar $\lambda \in L_{l o c}^{1}(X)$ such that

$$
-\int_{X}\langle\nabla \psi, \nabla(v \circ \varphi)\rangle=\int_{X} \psi \lambda\left[\left(\Delta_{N} v\right) \circ \varphi\right]
$$

for every $v \in \mathcal{C}^{2}(N)$ and $\psi \in \operatorname{Lip}_{c}(X)$ ( or $\psi \in W_{0}^{1,2}(X) \cap L^{\infty}(X)$ ).

In the affirmative case, $\lambda$ from the last equality equals a.e. the dilation of $\varphi$ (as a horizontally weakly conformal map).

As a consequence, for a harmonic morphism $\varphi: X \rightarrow N$, if $\psi: N \rightarrow P$ is a harmonic map between smooth Riemannian manifolds without boundary, then the composition $\psi \circ \varphi$ is a harmonic map.

\section{Pseudo-horizontally weakly conformal maps on Rie- mannian polyhedra.}

The aim of the present section, is to extend the notion of pseudo-horizontally weakly conformal maps on Riemannian manifolds (see [13]) to Riemannian polyhedra and to establish their properties. We will use the same terminology as in [13].

Let $(X, g)$ be an admissible Riemannian polyhedron of $\operatorname{dim} X=m$ and $\left(N, J^{N}, g_{N}\right)$ a Hermitian manifold of $\operatorname{dim}_{\mathbf{R}} N=2 n$, without boundary.

We denote by $\operatorname{Holom}(N)=\{f: N \rightarrow \mathbf{C}, f$ local holomorphic function $\}$. In what follows, the gradient operator and the inner product in $(X, g)$ are well defined a.e. in $X$ and will be denoted by $\nabla$ and $\langle$,$\rangle respectively.$

Definition 3.1 Let $\varphi: X \rightarrow N$ be a continuous map of class $W_{\text {loc }}^{1,2}(X, N)$. $\varphi$ is called pseudo-horizontally weakly conformal (shortening PHWC), iffor any pair of local holomorphic functions $v, w \in \operatorname{Holom}(N)$, such that $v=v_{1}+i v_{2}, w=w_{1}+i w_{2}$, we have:

$$
\begin{cases}\left\langle\nabla\left(w_{1} \circ \varphi\right), \nabla\left(v_{1} \circ \varphi\right)\right\rangle-\left\langle\nabla\left(w_{2} \circ \varphi\right), \nabla\left(v_{2} \circ \varphi\right)\right\rangle=0 & \text { a.e. in } X \\ \left\langle\nabla\left(w_{2} \circ \varphi\right), \nabla\left(v_{1} \circ \varphi\right)\right\rangle+\left\langle\nabla\left(w_{1} \circ \varphi\right), \nabla\left(v_{2} \circ \varphi\right)\right\rangle=0 & \text { a.e. in } X\end{cases}
$$

Remark 3.2 Definition 3.1 is a local one, hence it is sufficient to check the identities (1) in local complex coordinates $\left(z_{1}, z_{2}, \ldots, z_{n}\right)$ in $N$. Taking $z_{A}=x_{A}+i y_{A}, \forall A=1, \ldots, n$, 
the relations ([1), $\forall A, B=1, \ldots, n$, read:

$$
\left\{\begin{array}{cl}
\left\langle\nabla \varphi_{1}^{B}, \nabla \varphi_{1}^{A}\right\rangle-\left\langle\nabla \varphi_{2}^{B}, \nabla \varphi_{2}^{A}\right\rangle=0 & \text { a.e. in } X \\
\left\langle\nabla \varphi_{2}^{B}, \nabla \varphi_{1}^{A}\right\rangle+\left\langle\nabla \varphi_{1}^{B}, \nabla \varphi_{2}^{A}\right\rangle=0 & \text { a.e. in } X
\end{array}\right.
$$

where

$$
\left\{\begin{array}{l}
\varphi_{1}^{A}:=x_{A} \circ \varphi, \varphi_{2}^{A}:=y_{A} \circ \varphi, \quad \forall A=1, \ldots, n, \\
\varphi_{1}^{B}:=x_{B} \circ \varphi, \varphi_{2}^{B}:=y_{B} \circ \varphi, \quad \forall B=1, \ldots, n .
\end{array}\right.
$$

Remark 3.3 Definition 3.1 is justified by seeing that if the source manifold is a smooth Riemannian one, without boundary, we obtain exactly the commuting condition between $d \varphi_{x} \circ d \varphi_{x}^{*}$ and $J_{\varphi(x)}^{N}$ (see [13] or [2], [1]), where $d \varphi_{x}^{*}: T_{\varphi(x)} N \rightarrow T_{x} X$ is the adjoint map of the tangent linear map $d \varphi_{x}: T_{x} X \rightarrow T_{\varphi(x)} N$, for any $x \in X$.

The next proposition justifies the use of the term 'horizontally weakly conformal', indeed we obtain, when the target dimension is one, an equivalence between the horizontally weakly conformality $\mathrm{m}$ and pseudo-horizontally weakly conformality, as in the smooth case.

Proposition 3.4 Let $\varphi: X \rightarrow N$ a horizontally weakly conformal map (see subsection 2.3.2) from a Riemannian admissible polyhedron $(X, g)$ into a Hermitian manifold $\left(N, J^{N}, g_{N}\right)$. Then $\varphi$ is pseudo-horizontally weakly conformal. If the complex dimension of $N$ is equal to one, then the two conditions are equivalent.

Proof: Let $\varphi: X \rightarrow N$ be a horizontally weakly conformal map from an admissible Riemannian polyhedron $(X, g)$ into a Hermitian manifold $\left(N, J^{N}, g_{N}\right)$ of real dimension $2 n$. Take $\left(z_{A}=x_{A}+i y_{A}\right)_{A=1, \ldots, n}$ local complex coordinates in $N$. Then $\left\{\frac{\partial}{\partial x_{1}}, \ldots, \frac{\partial}{\partial x_{n}}, \frac{\partial}{\partial y_{1}}, \ldots, \frac{\partial}{\partial y_{n}}\right\}$ is a local frame in $T N$ such that

$$
\left\{\begin{array}{c}
J^{N}\left(\frac{\partial}{\partial x_{A}}\right)=\frac{\partial}{\partial y_{A}} \\
J^{N}\left(\frac{\partial}{\partial y_{A}}\right)=-\frac{\partial}{\partial x_{A}}
\end{array}, \forall A=1, \ldots, n\right.
$$

The horizontally weakly conformal condition reads in the considered frame:

$$
\left\langle\nabla \varphi^{\alpha}, \nabla \varphi^{\beta}\right\rangle=\lambda\left(g_{N}^{\alpha \beta} \circ \varphi\right) \text { a.e. in } X, \forall \alpha, \beta=1, \ldots, 2 n \text {. }
$$

where $\varphi^{\alpha}=\xi_{\alpha} \circ \varphi$ for $\xi_{\alpha}=\left\{\begin{array}{cc}x_{\alpha}, & \alpha=1, \ldots, n \\ y_{\alpha-n}, & \alpha=n+1, \ldots, 2 n\end{array}\right.$. Explicitly the equalities (3) are the following:

$$
\left\{\begin{array}{cc}
\left\langle\nabla\left(\xi_{\alpha} \circ \varphi\right), \nabla\left(\xi_{\beta} \circ \varphi\right)\right\rangle=\lambda\left[g_{N}\left(\frac{\partial}{\partial \xi_{\alpha}}, \frac{\partial}{\partial \xi_{\beta}}\right) \circ \varphi\right] & \text { a.e. in } X, \\
\left\langle\nabla\left(\xi_{\alpha} \circ \varphi\right), \nabla\left(\xi_{\beta} \circ \varphi\right)\right\rangle=\lambda\left[g_{N}\left(\frac{\partial}{\partial \xi_{\alpha}}, \frac{\partial}{\partial \xi_{\beta}}\right) \circ \varphi\right] & \forall \alpha, \beta=1, \ldots, n . \\
& \text { a.e. in } X, \\
& \forall \alpha, \beta=n+1, \ldots, 2 n .
\end{array}\right.
$$


and

$$
\left\{\begin{array}{cc}
\left\langle\nabla\left(\xi_{\alpha} \circ \varphi\right), \nabla\left(\xi_{\beta} \circ \varphi\right)\right\rangle=\lambda\left[g_{N}\left(\frac{\partial}{\partial \xi_{\alpha}}, \frac{\partial}{\partial \xi_{\beta}}\right) \circ \varphi\right] & \text { a.e. in } X, \\
& \forall \alpha=1, \ldots, n ; \\
& \forall \beta=n+1, \ldots, 2 n . \\
\left\langle\nabla\left(\xi_{\alpha} \circ \varphi\right), \nabla\left(\xi_{\beta} \circ \varphi\right)\right\rangle=\lambda\left[g_{N}\left(\frac{\partial}{\partial \xi_{\alpha}}, \frac{\partial}{\partial \xi_{\beta}}\right) \circ \varphi\right] & \text { a.e. in } X, \\
& \forall \alpha=n+1, \ldots, 2 n ; \\
& \forall \beta=1, \ldots, n .
\end{array}\right.
$$

Read (4) and (5) in terms of $x_{A}$ and $y_{B}, \forall A, B=1, \ldots, n$ :

$$
\left\{\begin{array}{cc}
\left\langle\nabla\left(x_{A} \circ \varphi\right), \nabla\left(x_{B} \circ \varphi\right)\right\rangle=\lambda\left[g_{N}\left(\frac{\partial}{\partial x_{A}}, \frac{\partial}{\partial x_{B}}\right) \circ \varphi\right] & \text { a.e. in } X, \\
\left\langle\nabla\left(y_{A} \circ \varphi\right), \nabla\left(y_{B} \circ \varphi\right)\right\rangle=\lambda\left[g_{N}\left(\frac{\partial}{\partial y_{A}}, \frac{\partial}{\partial y_{B}}\right) \circ \varphi\right] & \text { a.e. in } X .
\end{array}\right.
$$

and

$$
\left\{\begin{array}{cc}
\left\langle\nabla\left(x_{A} \circ \varphi\right), \nabla\left(y_{B} \circ \varphi\right)\right\rangle=\lambda\left[g_{N}\left(\frac{\partial}{\partial x_{A}}, \frac{\partial}{\partial y_{B}}\right) \circ \varphi\right] & \text { a.e. in } X, \\
\left\langle\nabla\left(y_{A} \circ \varphi\right), \nabla\left(x_{B} \circ \varphi\right)\right\rangle=\lambda\left[g_{N}\left(\frac{\partial}{\partial y_{A}}, \frac{\partial}{\partial x_{B}}\right) \circ \varphi\right] & \text { a.e. in } X .
\end{array}\right.
$$

Because $J^{N}$ is the complex structure with respect to the hermitian metric $g_{N}$, we have $\forall A, B=1, \ldots, n$ :

$$
\left\{\begin{array}{c}
g_{N}\left(\frac{\partial}{\partial x_{A}}, \frac{\partial}{\partial x_{B}}\right)=g_{N}\left(\frac{\partial}{\partial y_{A}}, \frac{\partial}{\partial y_{B}}\right) \\
g_{N}\left(\frac{\partial}{\partial x_{A}}, \frac{\partial}{\partial y_{B}}\right)=-g_{N}\left(\frac{\partial}{\partial y_{A}}, \frac{\partial}{\partial x_{B}}\right)
\end{array}\right.
$$

Invoking (8), (6) and (7) we conclude that $\varphi$ is pseudo-horizontally weakly conformal.

Consider now the case when $\operatorname{dim}_{\mathbf{C}} N=1$ and suppose $\varphi: X \rightarrow N$ is a pseudohorizontally weakly conformal map.

Let $z=x+i y$ be a local complex chart in $N$. In terms of this chart the pseudohorizontally weakly conformal condition (11) reads:

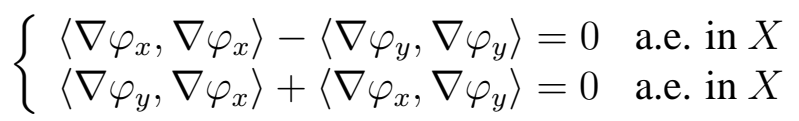

Remember that $g_{N}\left(\frac{\partial}{\partial x}, \frac{\partial}{\partial x}\right)=g_{N}\left(\frac{\partial}{\partial y}, \frac{\partial}{\partial y}\right) \neq 0$, so we can define

$$
\lambda:=\frac{\left\langle\nabla \varphi_{x}, \nabla \varphi_{x}\right\rangle}{g_{N}\left(\frac{\partial}{\partial x}, \frac{\partial}{\partial x}\right) \circ \varphi}=\frac{\left\langle\nabla \varphi_{y}, \nabla \varphi_{y}\right\rangle}{g_{N}\left(\frac{\partial}{\partial y}, \frac{\partial}{\partial y}\right) \circ \varphi}, \text { a.e. in } X
$$

From (9) and (10) we get:

$$
\left\{\begin{array}{cc}
\left\langle\nabla \varphi_{x}, \nabla \varphi_{x}\right\rangle=\lambda\left[g_{N}\left(\frac{\partial}{\partial x}, \frac{\partial}{\partial x}\right) \circ \varphi\right] & \text { a.e. in } X \\
\left\langle\nabla \varphi_{y}, \nabla \varphi_{y}\right\rangle=\lambda\left[g_{N}\left(\frac{\partial}{\partial y}, \frac{\partial}{\partial y}\right) \circ \varphi\right] & \text { a.e. in } X \\
\left\langle\nabla \varphi_{x}, \nabla \varphi_{y}\right\rangle=0 & \text { a.e. in } X
\end{array}\right.
$$

which means that $\varphi$ is horizontally weakly conformal. 
PHWC maps on Riemannian polyhedra can be characterized thanks to germs of holomorphic functions on the target Hermitian manifolds as follows:

Proposition 3.5 Let $\varphi: X \rightarrow N$ be a continuous map of class $W_{l o c}^{1,2}(X, N)$. Then $\varphi$ is pseudo-horizontally weakly conformal if and only if for any local holomorphic function $\psi: N \rightarrow \mathbf{C}, \psi \circ \varphi$ is also pseudo-horizontally weakly conformal.

Proof: Let $\varphi: X \rightarrow N$ be a continuous map of class $W_{l o c}^{1,2}(X, N)$, and $\psi: N \rightarrow \mathbf{C}$ be any holomorphic function with $\psi=\psi_{1}+i \psi_{2}$.

It is obvious (by definition) that if $\varphi$ is a PHWC map then $\psi \circ \varphi$ is a PHWC map.

Conversely, suppose now that for any holomorphic function $\psi: N \rightarrow \mathbf{C}$, the composition $\psi \circ \varphi: X \rightarrow \mathbf{C}$ is a PHWC function. Throughout the proof of Proposition 3.4 we have seen that this last fact reads:

$$
\begin{cases}\left\langle\nabla\left(\psi_{1} \circ \varphi\right), \nabla\left(\psi_{1} \circ \varphi\right)\right\rangle-\left\langle\nabla\left(\psi_{2} \circ \varphi\right), \nabla\left(\psi_{2} \circ \varphi\right)\right\rangle=0 & \text { a.e. in } X \\ \left\langle\nabla\left(\psi_{1} \circ \varphi\right), \nabla\left(\psi_{2} \circ \varphi\right)\right\rangle+\left\langle\nabla\left(\psi_{2} \circ \varphi\right), \nabla\left(\psi_{1} \circ \varphi\right)\right\rangle=0 & \text { a.e. in } X\end{cases}
$$

Then for a fixed local holomorphic chart $\left(z_{\alpha}\right)_{\alpha=1, \ldots, n}$ of $N, z_{\alpha}=x_{\alpha}+i y_{\alpha}$, the equalities (12) become:

$$
\left\{\begin{array}{c}
0=\left\langle\nabla\left(\psi_{1} \circ \varphi\right), \nabla\left(\psi_{1} \circ \varphi\right)\right\rangle-\left\langle\nabla\left(\psi_{2} \circ \varphi\right), \nabla\left(\psi_{2} \circ \varphi\right)\right\rangle= \\
\sum_{k, l=1}^{n}\left\langle\left(\partial_{k} \psi_{1} \circ \varphi\right) \cdot \nabla \varphi^{k},\left(\partial_{l} \psi_{1} \circ \varphi\right) \cdot \nabla \varphi^{l}\right\rangle- \\
\sum_{k, l=1}^{n}\left\langle\left(\partial_{k} \psi_{2} \circ \varphi\right) \cdot \nabla \varphi^{k},\left(\partial_{l} \psi_{2} \circ \varphi\right) \cdot \nabla \varphi^{l}\right\rangle \\
\text { and } \\
0=\left\langle\nabla\left(\psi_{1} \circ \varphi\right), \nabla\left(\psi_{2} \circ \varphi\right)\right\rangle+\left\langle\nabla\left(\psi_{2} \circ \varphi\right), \nabla\left(\psi_{1} \circ \varphi\right)\right\rangle= \\
\sum_{k, l=1}^{n}\left\langle\left(\partial_{k} \psi_{1} \circ \varphi\right) \cdot \nabla \varphi^{k},\left(\partial_{l} \psi_{2} \circ \varphi\right) \cdot \nabla \varphi^{l}\right\rangle+ \\
\sum_{k, l=1}^{n}\left\langle\left(\partial_{k} \psi_{2} \circ \varphi\right) . \nabla \varphi^{k},\left(\partial_{l} \psi_{1} \circ \varphi\right) \cdot \nabla \varphi^{l}\right\rangle \\
\text { where } \varphi^{\alpha}=\xi_{\alpha} \circ \varphi \text { for } \xi_{\alpha}=\left\{\begin{array}{c}
x_{\alpha}, \quad \alpha=1, \ldots, n \\
y_{\alpha-n}, \quad \alpha=n+1, \ldots, 2 n
\end{array} .\right.
\end{array}\right.
$$

Taking into account the Cauchy-Riemann equations and after doing some computations, (13) becomes: 


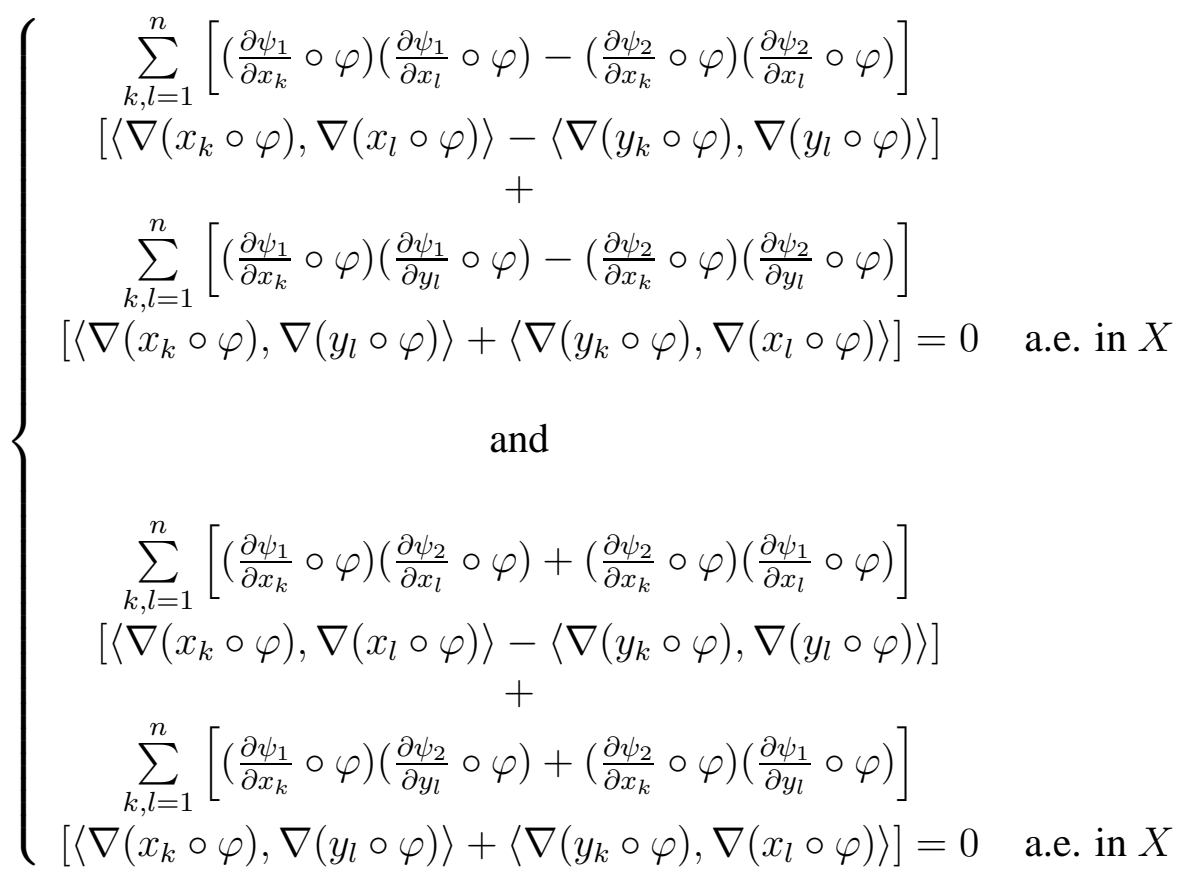

Now, choose particular holomorphic functions $\psi$ 's, for example locally $\psi=z_{k}+$ $z_{l}$ and vary the $k, l$ indices, we obtain all the pseudo-horizontally weakly conformality conditions of the map $\varphi$. This ends the proof.

The next proposition makes clear the relation between PHWC maps on Riemannian polyhedra and holomorphic maps on target Hermitian manifolds.

Proposition 3.6 Let $\varphi: X \rightarrow N$ a continuous map of class $W_{l o c}^{1,2}(X, N)$ and $\left(P, J^{P}, g_{P}\right)$ another Hermitian manifold of $\operatorname{dim}_{\mathbf{R}} \mathrm{P}=2 \mathrm{p}$. Then $\varphi$ is pseudo-horizontally weakly conformal if and only if for every local holomorphic map $\psi: N \rightarrow P, \psi \circ \varphi$ is also pseudo-horizontally weakly conformal.

Proof: Let $\psi: N \rightarrow P$ be a local holomorphic map. Choose $\left(z_{\alpha}\right)_{\alpha=1, \ldots, p}$ local complex coordinates in $P$ and denote $\psi^{\alpha}:=z_{\alpha} \circ \psi, \forall \alpha=1, \ldots, p$.

Suppose that $\varphi$ is pseudo-horizontally weakly conformal. Then, by definition we have, for every pair of local holomorphic functions $v, w \in \operatorname{Holom}(N)$, such that $v=v_{1}+i v_{2}$, $w=w_{1}+i w_{2}$,

$$
\begin{cases}\left\langle\nabla\left(w_{1} \circ \varphi\right), \nabla\left(v_{1} \circ \varphi\right)\right\rangle-\left\langle\nabla\left(w_{2} \circ \varphi\right), \nabla\left(v_{2} \circ \varphi\right)\right\rangle=0 & \text { a.e. in } X \\ \left\langle\nabla\left(w_{2} \circ \varphi\right), \nabla\left(v_{1} \circ \varphi\right)\right\rangle+\left\langle\nabla\left(w_{1} \circ \varphi\right), \nabla\left(v_{2} \circ \varphi\right)\right\rangle=0 & \text { a.e. in } X\end{cases}
$$

In particular, for every pair of local holomorphic functions on the Hermitian manifold $N$, $\psi^{\alpha}, \psi^{\beta}, \alpha, \beta=1, \ldots, p$ we have:

$$
\begin{cases}\left\langle\nabla\left(\psi_{1}^{\alpha} \circ \varphi\right), \nabla\left(\psi_{1}^{\beta} \circ \varphi\right)\right\rangle-\left\langle\nabla\left(\psi_{2}^{\alpha} \circ \varphi\right), \nabla\left(\psi_{2}^{\beta} \circ \varphi\right)\right\rangle=0 & \text { a.e. in } X \\ \left\langle\nabla\left(\psi_{2}^{\alpha} \circ \varphi\right), \nabla\left(\psi_{1}^{\beta} \circ \varphi\right)\right\rangle+\left\langle\nabla\left(\psi_{1}^{\alpha} \circ \varphi\right), \nabla\left(\psi_{2}^{\beta} \circ \varphi\right)\right\rangle=0 & \text { a.e. in } X\end{cases}
$$


have:

Or equivalently, if we denote $z_{\alpha}=x_{\alpha}+i y_{\alpha}$ and $\left\{\begin{array}{l}\psi_{1}^{\alpha}=x_{\alpha} \circ \psi \\ \psi_{2}^{\alpha}=y_{\alpha} \circ \psi\end{array}, \forall \alpha=1, \ldots, p\right.$, we

$$
\left\{\begin{array}{l}
\left\langle\nabla\left(x_{\alpha} \circ(\psi \circ \varphi)\right), \nabla\left(x_{\beta} \circ(\psi \circ \varphi)\right)\right\rangle- \\
\left\langle\nabla\left(y_{\alpha} \circ(\psi \circ \varphi)\right), \nabla\left(y_{\beta} \circ(\psi \circ \varphi)\right)\right\rangle=0 \quad \text { a.e. in } X \\
\left\langle\nabla\left(y_{\alpha} \circ(\psi \circ \varphi)\right), \nabla\left(x_{\beta} \circ(\psi \circ \varphi)\right)\right\rangle+ \\
\left\langle\nabla\left(x_{\alpha} \circ(\psi \circ \varphi)\right), \nabla\left(y_{\beta} \circ(\psi \circ \varphi)\right)\right\rangle=0 \quad \text { a.e. in } X
\end{array}\right.
$$

for every $\alpha, \beta=1, \ldots, p$. Which means by definition, that $\psi \circ \varphi$ is a PHWC map.

Conversely, suppose now that $\psi \circ \varphi$ is pseudo-horizontally weakly conformal for any local holomorphic map $\psi: N \rightarrow P$. Consider a local complex chart $\left(z_{\alpha}\right)_{\alpha=1, \ldots, p}$ in $P$. So the map $\psi^{\alpha} \circ \varphi, \forall \alpha=1, \ldots, p$ is pseudo-horizontally weakly conformal (in the sense that we apply (1) for $v=w=z_{\alpha}$ ).

In order to prove that $\varphi$ is a PHWC map we shall use Proposition 3.5. Let $u: N \rightarrow \mathbf{C}$ denote a local holomorphic function on $N$. We associate to $u$ a new map:

$$
\begin{aligned}
\phi_{u}: N & \rightarrow \\
x & \mapsto(0, \ldots, 0, \underbrace{u(x)}_{\alpha}, 0, \ldots, 0)
\end{aligned}
$$

For any local holomorphic chart $\eta: P \rightarrow \mathbf{C}^{p}$, we obtain a map $\psi_{u}: N \rightarrow P, \psi_{u}=$ $\eta^{-1} \circ \phi_{u}$ such that the $\alpha$ 's coordinate of $\psi_{u}$ is exactly the complex function $u: N \rightarrow \mathbf{C}$.

So we have proved that every local holomorphic function $u$ on $N$ can be obtained as a coordinate function of some local holomorphic map $\psi_{u}: N \rightarrow P$.

We have supposed before, that for every $\psi: N \rightarrow P$ and any local complex chart $\left(z_{\alpha}\right)_{\alpha=1, \ldots, p}$ in $P$,

$$
\begin{cases}\left\langle\nabla\left(\psi_{1}^{\alpha} \circ \varphi\right), \nabla\left(\psi_{1}^{\alpha} \circ \varphi\right)\right\rangle-\left\langle\nabla\left(\psi_{2}^{\alpha} \circ \varphi\right), \nabla\left(\psi_{2}^{\alpha} \circ \varphi\right)\right\rangle=0 & \text { a.e. in X } \\ \left\langle\nabla\left(\psi_{2}^{\alpha} \circ \varphi\right), \nabla\left(\psi_{1}^{\alpha} \circ \varphi\right)\right\rangle+\left\langle\nabla\left(\psi_{1}^{\alpha} \circ \varphi\right), \nabla\left(\psi_{2}^{\alpha} \circ \varphi\right)\right\rangle=0 & \text { a.e. in X }\end{cases}
$$

In particular, for $\psi=\psi_{u}\left(\psi^{\alpha}=u\right)$, we obtain $u \circ \varphi$ is pseudo-horizontally weakly conformal, for any $u \in \operatorname{Holom}(N)$. This implies (cf. Proposition 3.5) that $\varphi$ is a pseudohorizontally weakly conformal map.

\section{Pseudo harmonic morphism on Riemannian polyhedra.}

Similarly to the smooth case, if the target manifold is endowed with a Kähler structure one can enlarge the class of harmonic morphisms on Riemannian polyhedra to the class of pseudo harmonic morphisms.

Let $(X, g)$ denote an admissible Riemannian polyhedron and $\left(N, J^{N}, g_{N}\right)$ a Kähler manifold without boundary.

Definition 4.1 A map $\varphi: X \rightarrow N$ is called pseudo harmonic morphism (shortening PHM) if and only if $\varphi$ is a harmonic map ( in the sense of Korevaar-Schoen [11] and Eells-Fuglede [7]) and pseudo-horizontally weakly conformal. 
Now, we will give a characterization of a pseudo harmonic morphism in terms of the germs of the holomorphic functions on the target Kähler manifold, and the harmonic structure (in sense of Brelot, see ch 2 [7]) on the domain admissible Riemannian polyhedron.

Theorem 4.2 A continuous map $\varphi: X \rightarrow N$ of class $W_{\text {loc }}^{1,2}(X, N)$ is a pseudo harmonic morphism if and only if $\varphi$ pulls back local complex-valued holomorphic functions on $N$ to harmonic functions on $X$ (i.e. for any holomorphic function $\psi: V \rightarrow \mathbf{C}$ defined on a open subset $V$ of $N$ with $\varphi^{-1}(V)$ non-empty, the composition $\psi \circ \varphi: \varphi^{-1}(V) \rightarrow \mathbf{R}^{2}$ is harmonic if and only if $\varphi$ is $P H M$ ).

In order to prove the theorem, we shall need the following elementary lemma:

Lemma 4.3 Let $\varphi \in W_{l o c}^{1,2}(X, N)$ and $\psi \in \operatorname{Holom}(N)$. If $\psi=\psi^{1}+i \psi^{2}$, then $\psi \circ \varphi \in$ $W_{l o c}^{1,2}\left(X, \mathbf{R}^{2}\right)$ and moreover $\psi^{j} \circ \varphi \in W_{l o c}^{1,2}(X), \forall j=1,2$.

Proof: Let $\psi: N \rightarrow \mathbf{C}$ be a holomorphic function and $K \subset N$ denote a compact subset in $N$. We say that $\psi$ is uniformly Lipschitz in $K$ if there is a scalar $\lambda$ (depending on $K$ ), such that $\|\psi(p)-\psi(q)\| \leqslant \lambda d_{N}(p, q), \forall p, q \in K$, where $\|$.$\| denotes the usually norm in$ $\mathrm{C}$ and $d_{N}$ is the associated distance to the Riemannian metric $g_{N}$ on $N$. So $\psi$ is uniformly Lipschitz on $K$.

We take $U$ a quasiopen set on $X$ which is relatively compact. $\psi_{\mid \varphi(\bar{U})}$ is uniformly Lipschitz.

Now, following a result of Eells-Fuglede (see Corollary 9.1, p.158, [7])

$$
E_{U}(\psi \circ \varphi) \leqslant \lambda_{\varphi(\bar{U})}^{2} E_{U}(\varphi) .
$$

It suffices to cover $X$ by a countable quasiopen sets $U$, such that $E_{U}(\varphi)<\infty$ (such thing is possible because $\left.\varphi \in W_{l o c}^{1,2}(X, N)\right)$. We conclude that $\psi \circ \varphi \in W_{l o c}^{1,2}\left(X, \mathbf{R}^{2}\right)$.

For $\psi^{j} \circ \varphi, \forall j=1,2$, just remark that $\psi^{j}, \forall j=1,2$ are locally Lipschitz, so by the same argument used for proving that $\psi \circ \varphi \in W_{l o c}^{1,2}\left(X, \mathbf{R}^{2}\right)$, we have $\psi^{j} \circ \varphi \in W_{l o c}^{1,2}(X)$, $\forall j=1,2$.

Remark 4.4 Let $f: N \rightarrow \mathrm{C}$ be a local holomorphic function defined on a complex manifold $N$. Take $\left(z_{1}, \ldots, z_{n}\right)$ a local holomorphic coordinates in $N$ and denote $z_{j}=$ $x_{j}+y_{j}, \forall j=1, \ldots, n$. If the manifold $N$ is Kähler and if we write $f=f^{1}+i f^{2}$, than we have the following equalities ("symmetries"):

$$
\frac{\partial^{2} f^{j}}{\partial x_{A} \partial y_{B}}=\frac{\partial^{2} f^{j}}{\partial x_{B} \partial y_{A}}, \forall j=1,2 ; \forall A, B=1, \ldots, n .
$$

\section{Proof of Theorem 4.2:}

$" \Rightarrow "$ :

Let $\varphi: X \rightarrow N$ be a continuous map of class $W_{l o c}^{1,2}(X, N)$. Suppose that $\varphi$ is pseudoharmonic morphism and let $\psi: N \rightarrow \mathbf{C}$ be any local holomorphic function, with $\psi=$ $\psi^{1}+i \psi^{2}$. Remark that $\psi \circ \varphi$ is a continuous map ( as a composite of two continuous maps). Moreover, by Lemma4.3, $\psi \circ \varphi \in W_{l o c}^{1,2}\left(X, \mathbf{R}^{2}\right)$. 
In order to prove that $\psi \circ \varphi$ is harmonic, (cf. subsection 2.3.1) it suffices to show that $\psi \circ \varphi$ is weakly harmonic (as a map with values in $\mathbf{R}^{2}$ ).

The fact that $\psi \circ \varphi$ is weakly harmonic reads in the unique (conformal) chart $\eta: \mathbf{R}^{2} \rightarrow$ $\mathbf{R}^{2}$ and for any quasiopen set $U \subset \varphi^{-1}\left(\mathbf{R}^{2}\right)$ of compact closure in $X$,

$$
\begin{gathered}
\int_{U}\left\langle\nabla f, \nabla\left(\psi^{i} \circ \varphi\right)\right\rangle d \mu_{g}= \\
=\int_{U} f \cdot\left(\mathbf{R}^{2} \Gamma_{\alpha \beta}^{i} \circ \varphi\right)\left\langle\nabla\left(\psi^{\alpha} \circ \varphi\right), \nabla\left(\psi^{\beta} \circ \varphi\right)\right\rangle d \mu_{g}
\end{gathered}
$$

for $i=1,2$ and every bounded function $f \in W_{c}^{1,2}(U)$.

In the case of $\mathbf{R}^{2}$ we know that $\mathbf{R}^{2} \Gamma_{\alpha \beta}^{i} \equiv 0$, for any $\alpha, \beta=1,2$, so the equations (18) become:

$$
\int_{U}\left\langle\nabla f, \nabla\left(\psi^{i} \circ \varphi\right)\right\rangle d \mu_{g}=0, \forall i=1,2 .
$$

Let us prove now (19).

Take a real chart $\left(x_{1}, \ldots, x_{n}, y_{1}, \ldots, y_{n}\right)$ in $N$, such that the complex associated chart $\left(z_{j}=x_{j}+i y_{j}\right)_{j=1, n}$ is holomorphic. Then for any chart domain $V \subset N$ and quasiopen set $U \subset \varphi^{-1}(V)$ of compact closure in $X$, and for any functions $v \in \mathcal{C}^{2}(V)$ and $f \in$ $W_{c}^{1,2}(U) \cap L^{\infty}(U)$ we have, (cf. [7] Remark 9.7):

$$
\int_{U}\langle\nabla f, \nabla(v \circ \varphi)\rangle d \mu_{g}=\int_{U}\left\langle\left[\left(\partial_{\alpha} v\right) \circ \varphi\right] \nabla f, \nabla \varphi^{\alpha}\right\rangle d \mu_{g},
$$

where $\partial_{\alpha}$ denotes the $\alpha$ 's partial derivative.

By partial integration,

$$
\begin{aligned}
\int_{U}\langle\nabla f, \nabla(v \circ \varphi)\rangle d \mu_{g}= & \int_{U}\left\langle\nabla\left(f .\left[\left(\partial_{\alpha} v\right) \circ \varphi\right]\right), \nabla \varphi^{\alpha}\right\rangle d \mu_{g}- \\
& \int_{U} f \cdot\left[\left(\partial_{\alpha} \partial_{\beta} v\right) \circ \varphi\right]\left\langle\nabla \varphi^{\alpha}, \nabla \varphi^{\beta}\right\rangle d \mu_{g}
\end{aligned}
$$

Recall that in the local coordinates $\left(x_{1}, \ldots, x_{n}, y_{1}, \ldots, y_{n}\right)$ in $N$, we have:

$$
v_{\alpha \beta}=\partial_{\alpha} \partial_{\beta} v-{ }^{N} \Gamma_{\alpha \beta}^{k} \partial_{k} v, \forall \alpha, \beta=1,2,
$$

(where $v_{\alpha \beta}$ are the second order covariant derivatives of $v$ ). Inserting (22) in the last integral on the righthand side of the equation (21) we obtain:

$$
\begin{aligned}
& \int_{U}\langle\nabla f, \nabla(v \circ \varphi)\rangle d \mu_{g}=\quad \int_{U}\left\langle\nabla\left(f .\left[\left(\partial_{\alpha} v\right) \circ \varphi\right]\right), \nabla \varphi^{\alpha}\right\rangle d \mu_{g}- \\
& \int_{U}^{U} f .\left(v_{\alpha \beta} \circ \varphi\right)\left\langle\nabla \varphi^{\alpha}, \nabla \varphi^{\beta}\right\rangle d \mu_{g}- \\
& \int_{U} f \cdot\left[\left(\partial_{k} v\right) \circ \varphi\right]\left({ }^{N} \Gamma_{\alpha \beta}^{k} \circ \varphi\right)\left\langle\nabla \varphi^{\alpha}, \nabla \varphi^{\beta}\right\rangle d \mu_{g}
\end{aligned}
$$

$\varphi$ is supposed harmonic so it is weakly harmonic. Consequently, the first and the third integral in the righthand side of 23) are equal, so:

$$
\int_{U}\langle\nabla f, \nabla(v \circ \varphi)\rangle d \mu_{g}=-\int_{U} f .\left(v_{\alpha \beta} \circ \varphi\right)\left\langle\nabla \varphi^{\alpha}, \nabla \varphi^{\beta}\right\rangle d \mu_{g} .
$$


Take now $v=\psi^{i}$. Then for $i=1,2$ we have:

$$
\int_{U}\left\langle\nabla f, \nabla\left(\psi^{i} \circ \varphi\right)\right\rangle d \mu_{g}=-\int_{U} f .\left(\psi_{\alpha \beta}^{i} \circ \varphi\right)\left\langle\nabla \varphi^{\alpha}, \nabla \varphi^{\beta}\right\rangle d \mu_{g} .
$$

We compute the righthand side of the last equality:

$$
\begin{gathered}
\int_{U} f \cdot\left(\psi_{\alpha \beta}^{i} \circ \varphi\right)\left\langle\nabla \varphi^{\alpha}, \nabla \varphi^{\beta}\right\rangle d \mu_{g}= \\
=\int_{U} f \cdot \sum_{A, B=1}^{n}\left(\frac{\partial^{2} \psi^{i}}{\partial x_{A} \partial x_{B}} \circ \varphi\right)\left\langle\nabla\left(x_{A} \circ \varphi\right), \nabla\left(x_{B} \circ \varphi\right)\right\rangle d \mu_{g}+ \\
\int_{U} f \cdot \sum_{A, B=1}^{n}\left(\frac{\partial^{2} \psi^{i}}{\partial x_{A} \partial y_{B}} \circ \varphi\right)\left\langle\nabla\left(x_{A} \circ \varphi\right), \nabla\left(y_{B} \circ \varphi\right)\right\rangle d \mu_{g}+ \\
\int_{U} f \cdot \sum_{A, B=1}^{n}\left(\frac{\partial^{2} \psi^{i}}{\partial y_{A} \partial x_{B}} \circ \varphi\right)\left\langle\nabla\left(y_{A} \circ \varphi\right), \nabla\left(x_{B} \circ \varphi\right)\right\rangle d \mu_{g}+ \\
\int_{U} f \cdot \sum_{A, B=1}^{n}\left(\frac{\partial^{2} \psi^{i}}{\partial y_{A} \partial y_{B}} \circ \varphi\right)\left\langle\nabla\left(y_{A} \circ \varphi\right), \nabla\left(y_{B} \circ \varphi\right)\right\rangle d \mu_{g} .
\end{gathered}
$$

For example, for $i=1$ we have:

$$
\begin{gathered}
\int_{U} f \cdot\left(\psi_{\alpha \beta}^{1} \circ \varphi\right)\left\langle\nabla \varphi^{\alpha}, \nabla \varphi^{\beta}\right\rangle d \mu_{g}= \\
\int_{U} f \cdot \sum_{A, B=1}^{n}\left(\frac{\partial^{2} \psi^{1}}{\partial x_{A} \partial x_{B}} \circ \varphi\right)\left\langle\nabla\left(x_{A} \circ \varphi\right), \nabla\left(x_{B} \circ \varphi\right)\right\rangle d \mu_{g}+ \\
\int_{U} f \cdot \sum_{A, B=1}^{n}\left(\frac{\partial^{2} \psi^{1}}{\partial x_{A} \partial y_{B}} \circ \varphi\right)\left\langle\nabla\left(x_{A} \circ \varphi\right), \nabla\left(y_{B} \circ \varphi\right)\right\rangle d \mu_{g}+ \\
\int_{U} f \cdot \sum_{A, B=1}^{n}\left(\frac{\partial^{2} \psi^{1}}{\partial y_{A} \partial x_{B}} \circ \varphi\right)\left\langle\nabla\left(y_{A} \circ \varphi\right), \nabla\left(x_{B} \circ \varphi\right)\right\rangle d \mu_{g}+ \\
\int_{U} f . \sum_{A, B=1}^{n}\left(\frac{\partial^{2} \psi^{1}}{\partial y_{A} \partial y_{B}} \circ \varphi\right)\left\langle\nabla\left(y_{A} \circ \varphi\right), \nabla\left(y_{B} \circ \varphi\right)\right\rangle d \mu_{g} .
\end{gathered}
$$

Inserting Cauchy-Riemann equations associated to $\psi_{1}$ and $\psi_{2}$ (because $\psi$ is holomorphic) in the second and third sums of the righthand side of the above equality, we obtain:

$$
\begin{gathered}
\int_{U} f \cdot\left(\psi_{\alpha \beta}^{1} \circ \varphi\right)\left\langle\nabla \varphi^{\alpha}, \nabla \varphi^{\beta}\right\rangle d \mu_{g}= \\
\int_{U} f \cdot \sum_{A, B=1}^{n}\left(\frac{\partial^{2} \psi^{1}}{\partial x_{A} \partial x_{B}} \circ \varphi\right)\left\langle\nabla\left(x_{A} \circ \varphi\right), \nabla\left(x_{B} \circ \varphi\right)\right\rangle d \mu_{g}+ \\
\int_{U} f \cdot \sum_{A, B=1}^{n}\left(-\frac{\partial^{2} \psi^{2}}{\partial x_{A} \partial x_{B}} \circ \varphi\right)\left\langle\nabla\left(x_{A} \circ \varphi\right), \nabla\left(y_{B} \circ \varphi\right)\right\rangle d \mu_{g}+ \\
\int_{U} f \cdot \sum_{A, B=1}^{n}\left(\frac{\partial^{2} \psi^{2}}{\partial y_{A} \partial y_{B}} \circ \varphi\right)\left\langle\nabla\left(y_{A} \circ \varphi\right), \nabla\left(x_{B} \circ \varphi\right)\right\rangle d \mu_{g}+ \\
\int_{U} f \cdot \sum_{A, B=1}^{n}\left(\frac{\partial^{2} \psi^{1}}{\partial y_{A} \partial y_{B}} \circ \varphi\right)\left\langle\nabla\left(y_{A} \circ \varphi\right), \nabla\left(y_{B} \circ \varphi\right)\right\rangle d \mu_{g}
\end{gathered}
$$


By easy computations we obtain:

$$
\begin{gathered}
\int_{U} f .\left(\psi_{\alpha \beta}^{1} \circ \varphi\right)\left\langle\nabla \varphi^{\alpha}, \nabla \varphi^{\beta}\right\rangle d \mu_{g}=\int_{U} f \cdot \sum_{A, B=1}^{n}\left[\left(\frac{\partial^{2} \psi^{1}}{\partial x_{A} \partial x_{B}} \circ \varphi\right)-\left(\frac{\partial^{2} \psi^{1}}{\partial y_{A} \partial y_{B}} \circ \varphi\right)\right] \\
{\left[\left\langle\nabla\left(x_{A} \circ \varphi\right), \nabla\left(x_{B} \circ \varphi\right)\right\rangle-\left\langle\nabla\left(y_{A} \circ \varphi\right), \nabla\left(y_{B} \circ \varphi\right)\right\rangle\right] d \mu_{g}-} \\
\int_{U} f \cdot \sum_{A, B=1}^{n}\left[\left(\frac{\partial^{2} \psi^{2}}{\partial x_{A} \partial x_{B}} \circ \varphi\right)-\left(\frac{\partial^{2} \psi^{2}}{\partial y_{A} \partial y_{B}} \circ \varphi\right)\right] \\
{\left[\left\langle\nabla\left(x_{A} \circ \varphi\right), \nabla\left(y_{B} \circ \varphi\right)\right\rangle+\left\langle\nabla\left(y_{A} \circ \varphi\right), \nabla\left(x_{B} \circ \varphi\right)\right\rangle\right] d \mu_{g}+} \\
\int_{U} f \cdot \sum_{A, B=1}^{n}\left[\left(\frac{\partial^{2} \psi^{1}}{\partial x_{A} \partial x_{B}} \circ \varphi\right)\left\langle\nabla\left(y_{A} \circ \varphi\right), \nabla\left(y_{B} \circ \varphi\right)\right\rangle+\right. \\
\left.\left(\frac{\partial^{2} \psi^{1}}{\partial y_{A} \partial y_{B}} \circ \varphi\right)\left\langle\nabla\left(x_{A} \circ \varphi\right), \nabla\left(x_{B} \circ \varphi\right)\right\rangle\right] d \mu_{g}+ \\
\int_{U} f \cdot \sum_{A, B=1}^{n}\left[\left(\frac{\partial^{2} \psi^{2}}{\partial x_{A} \partial x_{B}} \circ \varphi\right)\left\langle\nabla\left(y_{A} \circ \varphi\right), \nabla\left(x_{B} \circ \varphi\right)\right\rangle-\right. \\
\left.\left(\frac{\partial^{2} \psi^{2}}{\partial y_{A} \partial y_{B}} \circ \varphi\right)\left\langle\nabla\left(x_{A} \circ \varphi\right), \nabla\left(y_{B} \circ \varphi\right)\right\rangle\right] d \mu_{g} .
\end{gathered}
$$

Because the map $\varphi$ is supposed pseudo-horizontally weakly conformal, the first and the second integral of the righthand side in the last equality are zero, so:

$$
\begin{gathered}
\int_{U} f .\left(\psi_{\alpha \beta}^{1} \circ \varphi\right)\left\langle\nabla \varphi^{\alpha}, \nabla \varphi^{\beta}\right\rangle d \mu_{g}= \\
\int_{U} f . \sum_{A, B=1}^{n}\left[\left(\frac{\partial^{2} \psi^{1}}{\partial x_{A} \partial x_{B}} \circ \varphi\right)+\left(\frac{\partial^{2} \psi^{1}}{\partial y_{A} \partial y_{B}} \circ \varphi\right)\right]\left\langle\nabla\left(x_{A} \circ \varphi\right), \nabla\left(x_{B} \circ \varphi\right)\right\rangle d \mu_{g}+ \\
+\int_{U} f . \sum_{A, B=1}^{n}\left[\left(\frac{\partial^{2} \psi^{2}}{\partial x_{A} \partial x_{B}} \circ \varphi\right)+\left(\frac{\partial^{2} \psi^{2}}{\partial y_{A} \partial y_{B}} \circ \varphi\right)\right]\left\langle\nabla\left(x_{A} \circ \varphi\right), \nabla\left(y_{B} \circ \varphi\right)\right\rangle d \mu_{g}
\end{gathered}
$$

Now, by Remark (4.4) the last two sums are zero. So we obtain:

$$
\int_{U}\left\langle\nabla f, \nabla\left(\psi^{1} \circ \varphi\right)\right\rangle d \mu_{g}=0 .
$$

By a similar computation we can also prove:

$$
\int_{U}\left\langle\nabla f, \nabla\left(\psi^{2} \circ \varphi\right)\right\rangle d \mu_{g}=0 .
$$

Thus $\psi \circ \varphi$ is weakly harmonic and so, harmonic (cf. subsection 2.3.1).

\section{$" \Leftarrow "$ :}

Conversely, suppose $\psi \circ \varphi: X \rightarrow \mathbf{R}^{2}$ is harmonic for any $\psi \in \operatorname{Holom}(N)$. It is known that the harmonicity of $\psi \circ \varphi$ is equivalent (cf. subsection 2.3.1 and Lemma 4.3) to the weak harmonicity of $\psi \circ \varphi$. So, for a given chart $\eta: \mathbf{R}^{2} \rightarrow \mathbf{R}^{2}$, and for any quasiopen set $U \subset \varphi^{-1}\left(\mathbf{R}^{2}\right)$ of compact closure in $X$, the weak harmonicity reads:

$$
\int_{U}\left\langle\nabla f, \nabla\left(\psi^{i} \circ \varphi\right)\right\rangle d \mu_{g}=0,
$$

for $i=1,2$ and every function $f \in W_{c}^{1,2}(U)$, where $\psi=\psi_{1}+i \psi_{2}$ :. 
Now, choose local complex holomorphic coordinates in $N,\left(z_{1}, \ldots, z_{n}\right)$, where $z_{k}=$ $x_{k}+i y_{k}, \forall k=1, \ldots, n$. Take $\psi=z_{k}, \forall k=1, \ldots, n$, and denote

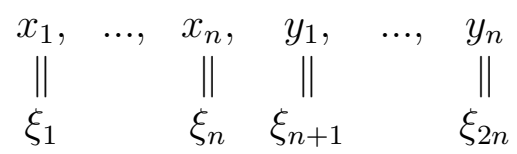

by a generic term $\xi^{k}, \forall k=1, \ldots, 2 n$. In particular, the equation (24), for the coordinates functions $\xi^{k}$, reads:

$$
\int_{U}\left\langle\nabla f, \nabla\left(\xi^{k} \circ \varphi\right)\right\rangle d \mu_{g}=0, \forall k=1, \ldots, 2 n .
$$

For the domain chart $V$ of $\left(z_{k}\right)$, any quasiopen set $U \subset \varphi^{-1}(V)$ of compact closure in $X$ and for $f \in W_{c}^{1,2}(U)$ we have:

$$
\begin{gathered}
0=\int_{U}\left\langle\nabla f, \nabla\left(\xi^{k} \circ \varphi\right)\right\rangle d \mu_{g}=\int_{U}\left\langle\nabla\left(f .\left[\left(\partial_{\alpha} \xi^{k}\right) \circ \varphi\right]\right), \nabla \varphi^{\alpha}\right\rangle d \mu_{g}- \\
\int_{U} f .\left(\xi_{\alpha \beta}^{k} \circ \varphi\right)\left\langle\nabla \varphi^{\alpha}, \nabla \varphi^{\beta}\right\rangle d \mu_{g}- \\
\int_{U} f \cdot\left[\left(\partial_{\gamma} \xi^{k}\right) \circ \varphi\right]\left({ }^{N} \Gamma_{\alpha \beta}^{\gamma} \circ \varphi\right)\left\langle\nabla \varphi^{\alpha}, \nabla \varphi^{\beta}\right\rangle d \mu_{g}, \\
\forall \alpha, \beta, \gamma=1, \ldots, 2 n .
\end{gathered}
$$

Taking into account that: $\forall k=1, \ldots, 2 n$ and $\forall \alpha, \beta=1, \ldots, 2 n$,

$$
\xi_{\alpha \beta}^{k}=0 \text { and } \partial_{\alpha} \xi^{k}=\left\{\begin{array}{ll}
0, & \text { if } \alpha=k \\
1, & \text { if } \alpha \neq k
\end{array},\right.
$$

we obtain:

$$
\begin{gathered}
0=\int_{U}\left\langle\nabla f, \nabla\left(\xi^{k} \circ \varphi\right)\right\rangle d \mu_{g}= \\
=\int_{U}\left\langle\nabla f, \nabla \varphi^{k}\right\rangle d \mu_{g}-\int_{U} f .\left({ }^{N} \Gamma_{\alpha \beta}^{k} \circ \varphi\right)\left\langle\nabla \varphi^{\alpha}, \nabla \varphi^{\beta}\right\rangle d \mu_{g},
\end{gathered}
$$

So, $\forall \alpha, \beta, k=1, \ldots, 2 n$,

$$
\int_{U}\left\langle\nabla f, \nabla \varphi^{k}\right\rangle d \mu_{g}=\int_{U} f .\left({ }^{N} \Gamma_{\alpha \beta}^{k} \circ \varphi\right)\left\langle\nabla \varphi^{\alpha}, \nabla \varphi^{\beta}\right\rangle d \mu_{g} .
$$

This means that $\varphi$ is weakly harmonic. But $\varphi$ is a continuous map of class $W_{l o c}^{1,2}(X, N)$, so it is a harmonic map (cf. subsection 2.3.1).

Now, for any $v: N \rightarrow \mathbf{R}$ such that $v \in \mathcal{C}^{2}(V)$, where $V$ is a domain chart on $N$, we have:

$$
\begin{aligned}
& \int_{U}\langle\nabla f, \nabla(v \circ \varphi)\rangle d \mu_{g}=\quad \int_{U}\left\langle\nabla\left(f\left[\left(\partial_{k} v\right) \circ \varphi\right]\right), \nabla \varphi^{k}\right\rangle d \mu_{g}- \\
& \int_{U} f\left(v_{\alpha \beta} \circ \varphi\right)\left\langle\nabla \varphi^{\alpha}, \nabla \varphi^{\beta}\right\rangle d \mu_{g}- \\
& \int_{U} f\left[\left(\partial_{k} v\right) \circ \varphi\right]\left({ }^{N} \Gamma_{\alpha \beta}^{k} \circ \varphi\right)\left\langle\nabla \varphi^{\alpha}, \nabla \varphi^{\beta}\right\rangle d \mu_{g}
\end{aligned}
$$


for $\alpha, \beta=1, \ldots, 2 n$, where $U \subset \varphi^{-1}(V)$ is quasiopen with compact closure in $X$.

In particular, for any holomorphic chart:

$$
\begin{array}{cl}
\eta: V \subset N & \rightarrow \mathbf{C}^{n} \\
p & \mapsto\left(z_{1}, \ldots, z_{n}\right), \quad z_{j}=x_{j}+i y_{j},
\end{array}
$$

and for any local holomorphic function $\psi: N \rightarrow \mathbf{C}, \psi=\psi^{1}+i \psi^{2}$, denoting $x_{1}, \ldots, x_{n}, y_{1}, \ldots, y_{n}$ by $\xi^{\gamma}, \forall \gamma=1, \ldots, 2 n$, we apply equation (26) to $\psi^{1}$ and $\psi^{2}$ respectively:

$$
\begin{aligned}
& 0=\quad \int_{U}\left\langle\nabla\left(f\left[\left(\partial_{k} \psi^{i}\right) \circ \varphi\right]\right), \nabla\left(\xi^{k} \circ \varphi\right)\right\rangle d \mu_{g}- \\
& \int_{U} f\left(\psi_{\alpha \beta}^{i} \circ \varphi\right)\left\langle\nabla\left(\xi^{\alpha} \circ \varphi\right), \nabla\left(\xi^{\beta} \circ \varphi\right)\right\rangle d \mu_{g}- \\
& \int_{U} f\left[\left(\partial_{k} \psi^{i}\right) \circ \varphi\right]\left({ }^{N} \Gamma_{\alpha \beta}^{k} \circ \varphi\right)\left\langle\nabla\left(\xi^{\alpha} \circ \varphi\right), \nabla\left(\xi^{\beta} \circ \varphi\right)\right\rangle d \mu_{g}, \quad \forall i=1,2 .
\end{aligned}
$$

In the above equality, the first and the last integral are equal (because $\varphi$ is harmonic), so we have:

$$
0=\int_{U} f\left(\psi_{\alpha \beta}^{i} \circ \varphi\right)\left\langle\nabla\left(\xi^{\alpha} \circ \varphi\right), \nabla\left(\xi^{\beta} \circ \varphi\right)\right\rangle d \mu_{g} .
$$

In the proof of the "if" part we have obtained:

$$
\begin{gathered}
0=\int_{U} f\left(\psi_{\alpha \beta}^{1} \circ \varphi\right)\left\langle\nabla\left(\xi^{\alpha} \circ \varphi\right), \nabla\left(\xi^{\beta} \circ \varphi\right)\right\rangle d \mu_{g}= \\
=\int_{U} f \cdot \sum_{A, B=1}^{n}\left[\left(\frac{\partial^{2} \psi^{1}}{\partial x_{A} \partial x_{B}} \circ \varphi\right)-\left(\frac{\partial^{2} \psi^{1}}{\partial y_{A} \partial y_{B}} \circ \varphi\right)\right] \\
{\left[\left\langle\nabla\left(x_{A} \circ \varphi\right), \nabla\left(x_{B} \circ \varphi\right)\right\rangle-\left\langle\nabla\left(y_{A} \circ \varphi\right), \nabla\left(y_{B} \circ \varphi\right)\right\rangle\right] d \mu_{g}-} \\
\int_{U} f \cdot \sum_{A, B=1}^{n}\left[\left(\frac{\partial^{2} \psi^{2}}{\partial x_{A} \partial x_{B}} \circ \varphi\right)-\left(\frac{\partial^{2} \psi^{2}}{\partial y_{A} \partial y_{B}} \circ \varphi\right)\right] \\
{\left[\left\langle\nabla\left(x_{A} \circ \varphi\right), \nabla\left(y_{B} \circ \varphi\right)\right\rangle+\left\langle\nabla\left(y_{A} \circ \varphi\right), \nabla\left(x_{B} \circ \varphi\right)\right\rangle\right] d \mu_{g}+} \\
\int_{U} f . \sum_{A, B=1}^{n}\left[\left(\frac{\partial^{2} \psi^{1}}{\partial x_{A} \partial x_{B}} \circ \varphi\right)\left\langle\nabla\left(y_{A} \circ \varphi\right), \nabla\left(y_{B} \circ \varphi\right)\right\rangle+\right. \\
\left.\left(\frac{\partial^{2} \psi^{1}}{\partial y_{A} \partial y_{B}} \circ \varphi\right)\left\langle\nabla\left(x_{A} \circ \varphi\right), \nabla\left(x_{B} \circ \varphi\right)\right\rangle\right] d \mu_{g}+ \\
\int_{U} f . \sum_{A, B=1}^{n}\left[\left(\frac{\partial^{2} \psi^{2}}{\partial x_{A} \partial x_{B}} \circ \varphi\right)\left\langle\nabla\left(y_{A} \circ \varphi\right), \nabla\left(x_{B} \circ \varphi\right)\right\rangle-\right. \\
\left.\left(\frac{\partial^{2} \psi^{2}}{\partial y_{A} \partial y_{B}} \circ \varphi\right)\left\langle\nabla\left(x_{A} \circ \varphi\right), \nabla\left(y_{B} \circ \varphi\right)\right\rangle\right] d \mu_{g} .
\end{gathered}
$$

Choosing particular holomorphic functions as: $\psi=z_{A} z_{B}$ and $\psi=i z_{A} z_{B}, \forall A, B=$ $1, \ldots, n$, in equation (27), we obtain the pseudo-horizontally weakly conformal conditions (see Definition 3.1).

A straightforward result deduced from the Theorem 4.2 is the folowing:

Corollary 4.5 Let $\varphi: X \rightarrow N$ be a pseudo-horizontally weakly conformal map from a Riemannian polyhedron into a Kähler manifold. Then $\varphi$ is harmonic if and only if the components $\varphi^{k}=z_{k} \circ \varphi$ in terms of any holomorphic coordinates $\left(z_{k}\right)_{k=1, \ldots, n}$ in $N$, are harmonic maps ( $\varphi^{k}$ is understood as a map into $\mathbf{R}^{2}$ ).

Proof: The "only if part" is already proved through the proof of the Theorem 4.2.

The "if part": Suppose that the map $\varphi$ is harmonic. So it is pseudo harmonic morphism. By Theorem 4.2, $\varphi$ pulls back local holomorphic functions to local harmonic maps which applies to any holomorphic coordinates in $N$. 
We turn our attention to the relation between pseudo harmonic morphism on Riemannian polyhedron and local holomorphic maps between Kähler manifolds. More precisely, we have:

Theorem 4.6 Let $(X, g)$ be a Riemannian polyhedron and $\left(N, J^{N}, g_{N}\right),\left(P, J^{P}, g_{P}\right)$ be two Kähler manifolds with $\operatorname{dim}_{\mathbf{C}} N=n$ and $\operatorname{dim}_{\mathbf{C}} P=p$. A continuous map $\varphi: X \rightarrow N$, of class $W_{\text {loc }}^{1,2}(X, N)$, is pseudo harmonic morphism if and only if $\psi \circ \varphi: X \rightarrow P$ is a (local) pseudo harmonic morphism for all local holomorphic maps $\psi: N \rightarrow P$.

Proof: $" \Rightarrow "$ :

Suppose that $\varphi: X \rightarrow N$ is a pseudo harmonic morphism and let $\psi: X \rightarrow P$ be any local holomorphic map.

By Proposition 3.6, $\psi \circ \varphi$ is a pseudo-horizontally weakly conformal map.

Let $\left(z_{1}, \ldots, z_{p}\right)$ be local holomorphic coordinates in $P$, and set $\psi^{j}=z_{j} \circ \psi$. On one hand, the map $\varphi$ is supposed PHM and obviously the complex functions $\psi^{j}: P \rightarrow \mathbf{C}$, $\forall j=1, \ldots, p$, are holomorphic so, by Theorem 4.2, $\psi^{j} \circ \varphi$ are harmonic, $\forall j=1, \ldots, p$.

On the other hand, $\psi \circ \varphi$ is pseudo-horizontally weakly conformal. Thus by Corollary 4.5, the map $\psi \circ \varphi$ is (local) harmonic.

$" \Leftarrow "$ :

Suppose now that $\psi \circ \varphi: X \rightarrow P$ is a (local) pseudo harmonic morphism, for any local holomorphic map $\psi: N \rightarrow P$. By Proposition 3.6 the map $\varphi$ is pseudo-horizontally weakly conformal.

As we have already done in the proof of Proposition 3.6. every local holomorphic function $v: N \rightarrow \mathbf{C}$ can be obtained as a coordinate of some local holomorphic map $\psi_{v}: N \rightarrow P$.

$\psi_{v} \circ \varphi$ is local a pseudo harmonic morphism. By Corollary $4.5 v \circ \varphi$ is harmonic (because it is a coordinate of $\psi_{v} \circ \varphi$ ). Therefore, we have shown that for any local holomorphic function $v: N \rightarrow \mathbf{C}$, the map $v \circ \varphi$ is harmonic. So by Theorem 4.2 we conclude that $\varphi$ is a pseudo harmonic morphism.

The following proposition will play an important roll in the next section for constructing examples of PHM on admissible Riemannian polyhedra.

Proposition 4.7 Let $(X, g)$ and $(Y, h)$ be two admissible Riemannian polyhedra, and $\left(N, J^{N}, g_{N}\right)$ a Kähler manifold without boundary of complex dimension n. Let $\varphi: Y \rightarrow$ $N$ be a continuous map of class $W_{l o c}^{1,2}(Y, N), \pi: X \rightarrow Y$ a proper, surjective, continuous map of class $W_{l o c}^{1,2}(X, Y)$ and $\widetilde{\varphi}=\varphi \circ \pi$. If $\pi$ is a harmonic morphism, then $\varphi$ is pseudo harmonic morphism if and only if $\widetilde{\varphi}$ is pseudo harmonic morphism.

Proof: Suppose that $\widetilde{\varphi}: X \rightarrow N$ is a pseudo harmonic morphism; by Theorem 4.2 it is equivalent to the fact that $\widetilde{\varphi}$ pulls back local holomorphic functions on $N$ to local harmonic functions on $X$. So, for any $\psi \in \operatorname{Holom}(N), \psi=\psi_{1}+i \psi_{2}$, the map $\psi \circ \widetilde{\varphi} \in$ $W_{l o c}^{1,2}\left(X, \mathbf{R}^{2}\right)$ is locally harmonic. But this can also reads: for any $\psi \in \operatorname{Holom}(N)$, $\psi=\psi_{1}+i \psi_{2}$, the functions $\psi_{1} \circ \widetilde{\varphi}$ and $\psi_{2} \circ \widetilde{\varphi}$ are locally harmonic (because $\psi \circ \widetilde{\varphi}$ is continuous and the Christoffel symbols relative to the fixed chart of $\mathbf{R}^{2}$ are all zero [cf. subsection 2.3.1]); or, also for any $\psi \in \operatorname{Holom}(N)$, with $\psi=\psi_{1}+i \psi_{2}$, the functions $\left(\psi_{1} \circ \varphi\right) \circ \pi$ and $\left(\psi_{2} \circ \varphi\right) \circ \pi$ are locally harmonic. 
On the other hand, the map $\pi$ is a harmonic morphism and it is supposed surjective and proper so, by subsection 2.3.2 (cf. [7], Theorem 13.1), for every open set $V \subset Y$ and any function $v: V \rightarrow \mathbf{R}$, the function $v$ is harmonic if and only if $v \circ \pi$ is harmonic. In particular this fact applies to the functions $\left(\psi_{1} \circ \varphi\right)$ and $\left(\psi_{2} \circ \varphi\right)$; in other words, for any local holomorphic function $\psi=\psi_{1}+i \psi_{2}$ on $N$, the functions $\left(\psi_{1} \circ \varphi\right) \circ \pi$ respectively $\left(\psi_{2} \circ \varphi\right) \circ \pi$ are locally harmonic if and only if the functions $\left(\psi_{1} \circ \varphi\right)$ respectively $\left(\psi_{2} \circ \varphi\right)$ are locally harmonic.

The last assertion is equivalent to the fact that, for any $\psi \in \operatorname{Holom}(N)$, the map $\psi \circ \varphi$ is locally harmonic. But by Theorem 4.2 this means (iff) that $\varphi: Y \rightarrow N$ is a pseudo harmonic morphism. This ends the proof of the proposition.

\section{Some examples.}

In this short section we will offer some examples of pseudo harmonic morphisms on Riemannian polyhedra.

As we have shown in Proposition 3.4 every horizontally weakly conformal map from a Riemannian admissible polyhedron into a Hermitian manifold, is pseudo horizontally weakly conformal. So every harmonic morphism into a Kähler manifold is pseudo harmonic morphism. For other nontrivial examples, when the source polyhedra are smooth Riemannian manifolds, see for example [1].

Thanks to Proposition 4.7 we can derive a several non-obvious examples of pseudo harmonic morphisms on (singular) Riemannian polyhedra.

It is known (see Example 8.12, [7]) that given a $K$ compact group of isometries of a complete smooth Riemannian manifold $M$, and $\pi: M \rightarrow M / K$ the projection onto the orbit space $M / K$ (with the quotient topology), there is a smooth triangulation of $M$ for which $\pi$ induces an admissible Riemannian polyhedral structure on $M / K$. The associated intrinsic distance $d_{M / K}\left(y_{1}, y_{2}\right)$ between elements $y_{1}, y_{2}$ of $M / K$, equals the intrinsic distance in $M$ between the corresponding compact orbits $\pi^{-1}\left(y_{1}\right)$ and $\pi^{-1}\left(y_{2}\right)$, and the polyhedron $M / K$ with the distance $d_{M / K}$ is a geodesic space. The polyhedral structure determines a Brelot harmonic sheaf $\mathcal{H}_{M / K}$ on $M / K$ (cf. Theorem 7.1, [7]). Moreover, $\pi$ caries the Brelot harmonic sheaf $\mathcal{H}_{M}$ onto a Brelot harmonic sheaf $\mathcal{H}_{M / K}^{\prime}=\pi_{*} \mathcal{H}_{M}$ on $M / K$ and $\pi:\left(M, \mathcal{H}_{M}\right) \rightarrow\left(M / K, \mathcal{H}_{M / K}^{\prime}\right)$ becomes a harmonic morphism, surjective and proper.

This construction can be applied to Riemannian orbifolds (cf. Subexample 8.13(ii), [7]) as follows:

Let $M$ be a Riemannian manifold, and $\mathcal{S}^{r}$ the symmetry group on $r$ factors.

Denote:

$$
\mathcal{S}^{r} \mathcal{M}:=(\underbrace{M \times M \times \ldots \times M}_{r \text {-times }}) / \mathcal{S}^{r}
$$

the $r$-fold symmetric power of the manifold $M$.

The compact group $\mathcal{S}^{r}$ acts isometrically, so $\mathcal{S}^{r} \mathcal{M}$ becomes a Riemannian orbifold (singular if the dimension of $M \geqslant 3$ ). Thus, as above, we obtain a proper, surjective, harmonic morphism from $M \times M \times \ldots \times M$ to $\mathcal{S}^{r} \mathcal{M}$. 
In the particular case when $M=\mathbf{C}^{k+s}$, with $2(k+s) \geqslant 3$, following [1], one can construct a pseudo harmonic morphism:

$$
\begin{aligned}
\eta: \mathbf{C}^{k} \times \mathbf{C}^{s} & \rightarrow & \mathbf{C}^{r} & \text { given by } \\
(u, v) & \mapsto & \left(\frac{F_{1}(u) P_{1}(\bar{v})}{G_{1}(u) Q_{1}(\bar{v})}, \ldots, \frac{F_{r}(u) P_{r}(\bar{v})}{G_{r}(u) Q_{r}(\bar{v})}\right) &
\end{aligned}
$$

where $F_{1}, \ldots, F_{r}, G_{1}, \ldots, G_{r}$ are homogenous polynomials on $\mathbf{C}^{k}$ and $P_{1}, \ldots, P_{r}, Q_{1}, \ldots, Q_{r}$ are homogenous polynomials on $\mathrm{C}^{s}$, all having the same degree. We know that the sum of two PHM is also a PHM, so we define:

$$
\widetilde{\varphi}: \mathbf{C}^{k+s} \times \mathbf{C}^{k+s} \rightarrow \mathbf{C}^{r}, \widetilde{\varphi}(\widetilde{u}, \widetilde{v}):=\eta(\widetilde{u})+\eta(\widetilde{v}),
$$

for any $\widetilde{u}, \widetilde{v} \in \mathbf{C}^{k+s}$.

Using the harmonic morphism $\mathbf{C}^{k+s} \times \mathbf{C}^{k+s} \rightarrow \mathcal{S}^{2} \mathcal{C}^{k+s}$, the map $\widetilde{\varphi}$ factors through a PHM from $\mathcal{S}^{2} \mathcal{C}^{k+s}$ to $\mathrm{C}^{r}$.

Moreover, using the harmonic morphism $\mathbf{C}^{k+s} \rightarrow \mathbf{C} P^{k+s-1}$, the map $\eta$ factors (see [1]) through a PHM from $\mathbf{C} P^{k+s-1}$ to $\mathbf{C}^{r}$ which is neither holomorphic nor antiholomorphic. Now using this map and apply the same arguments as before, we get a pseudo harmonic morphism from $\mathcal{S}^{2} \mathcal{C} \mathcal{P}^{k+s-1}$ to $\mathbf{C}^{r}$.

Acknowledgments. We thank the ICTP Trieste for hospitality during this work.

\section{References}

[1] M. A. Aprodu, M. Aprodu, V. Brînzănescu: A class of harmonic submersions and minimal submanifolds, Int. J. Math., 11, no.9, 1177-1191 (2000).

[2] P.Baird, J.C.Wood: Harmonic Morphisms Between Riemannian Manifolds, Oxford University Press (2003).

[3] W. Ballmann, M. Brin: Orbihedra of Nonpositive Curvature, Publications IHES, 82, 169-209 (1995).

[4] M.R. Bridson: Geodesics and Curvature in Metric Simplicial Complexes, World Scientific, Eds. E. Ghys, A.Haefliger, A. Verjovsky (1990).

[5] M.R. Bridson, A. Haefliger: Metric spaces of Non-positive curvature, SpringerVerlag (1999).

[6] M. Davis, T. Januszkiewicz: Hyperbolization of polyhedra, Journal of Differential Geometry, 34, no. 2, 347-388 (1991).

[7] J. Eells, B. Fuglede: Harmonic maps between Riemannien Polyhedra, Cambridge University Press (2001).

[8] B. Fuglede: Harmonic morphisms between Riemannian manifolds, Ann. Inst. Fourier 28, 107-144 (1978). 
[9] B. Giesecke: Simpliziale Zerlegung abzählbarer analytischer Räume, Math. Z. 83, 177-213 (1964).

[10] T. Ishihara: A mapping of Riemannian manifolds which preserves harmonic functions, J. Math. Kyoto Univ.(JMKYAZ) 19, 215-229 (1979).

[11] N. J. Korevaar, R. M. Schoen: Sobolev spaces and harmonic maps for metric space targets, Comm. Anal. Geom. 1, no. 3-4, 561-659 (1993).

[12] L. Lemaire: Applications harmoniques de surfaces riemanniennes, J. Diff. Geom. 13, 51-78 (1978).

[13] E.Loubeau: Pseudo Harmonic Morphisms, Int. J. Math. 7, 943-957 (1997).

[14] C. B. Morrey: The problem of plateau on a riemannian manifold, Ann. of Math. 149, 807-851 (1948).

[15] E. H. Spanier: Algebraic Topology, McGraw-Hill, New York (1966).

[16] J. Tits: Buildings of spherical type and finite BN-pairs, Lect. Notes in Math., vol. 386, Springer-Verlag (1974).

[17] H. Urakawa: Calculus of variations and harmonic maps, Transl. of Math. Monographs, 132, AMS, Providence (1993).

[18] Y. Xin: Geometry of Harmonic Maps, Progress in Nonlinear Diff. Eq. and their Appl., 23, 121-132, Birkhäuser Boston (1996). 